\title{
Discussion of Current Distribution of Exercise 3D MCG Using an Inhomogeneous Human Volume Conductor
}

\author{
M. De Melis, B.S. Kim, Y. Uchikawa*, H.-P. Müller**, S.N. Erné ${ }^{* * *}$ \\ School of science and engineering, Tokyo Denki Univ., Ishizaka, Hatoyama, Hiki-gun, Saitama 350-0394, Japan \\ *Graduate school of science and engineering, Tokyo Denki Univ., Ishizaka, Hatoyama, Hiki-gun, Saitama 350-0394, Japan \\ ${ }^{* *}$ HPM-Physics and software, Alpspitzweg 15, Neu Ulm 89231, Germany \\ ${ }^{* * *}$ Clinic of Neurology, Friedrich Schiller Univ., Erlangen Allee 101, Jena 07747, Germany \\ ${ }^{* * *}$ BMDSys Gmbh, Wildenbruchstrasse 15, Jena 07745, Germany
}

In this paper we present the analysis of 3D measurements of the magnetocardiogram by means of current source reconstruction. The data considered are rest and exercise stress data of test subjects. The source model used to describe the heart activation is a distributed source model, which allows to follow how the heart activation spreads over the myocardium. We developed a four compartments human torso model including the heart cavity and two lungs. Different values of the compartment conductivities are used to study the effect of the inhomogeneities on the reconstructed sources. The comparison between rest and stress data is done by means of correlations coefficients that indicate how close the reconstructed sources are in terms of dipoles moments, since in the source model considered the dipoles positions are fixed and depend on the source space model geometry. The same analyses are repeated using the 3D magnetic data and only the normal component, to investigate the additional information given by the tangential components. The results show that the torso and heart conductivities have a stronger influence than the one of the lungs, and that the $3 \mathrm{D}$ data are less sensitive to the changes of the compartment conductivities.

Key words: magnetocardiogram (MCG), stress exercise test, inverse problem, torso model, conductivity, correlation.

\section{Introduction}

Current source reconstruction (CSR) of the magnetocardiogram (MCG) signals is a useful tool to visualize the current flow in the human heart. The magnetic signals recorded by multichannel MCG systems are used in order to compute the solution of the so-called inverse problem, thus obtaining a reconstructed source that can be used to evaluate the electric activity in the myocardium. Such a method can be applied to study the electric activation in patients that suffered from myocardial infarction (MI) or affected by coronary artery disease (CAD). A common method of analysis for CAD and MI patients is exercise testing to provoke ischemia ${ }^{1)-2)}$. The MCG system used is able to detect three-dimensional (3D) magnetic fields. Simulation studies to evaluate the information gain obtained using vector fields have been carried out ${ }^{3)}$. The aim of the present study is to apply CSR on exercise test measurements to select a criterion of comparison, specifically correlation coefficients, between rest and stress data so that their differences can be quantified and to investigate the influence of the torso inhomogeneities on these coefficients. For this purpose we developed a volume conductor model that includes the torso, the heart cavity and the lungs. The analyses were repeated using the $3 \mathrm{D}$ components data and only the normal component of the same subjects in order to show what is the contribution given by the tangential components of the magnetic fields in the calculation of the CSR with real data. All data analyses were performed using the software MFIView of the BMDSys Gmbh (Jena, Germany).

\section{MCG measurements}

The MCG measurements were carried out on the MCG system at the Tokyo Denki University (TDU)4)-5), that operates inside a manetically shielded room (MSR), using a second order gradiometer shown in Fig. 1, which was orthogonal wound on a rectungular solid $3 \times 3 \times 6 \mathrm{~cm}$.

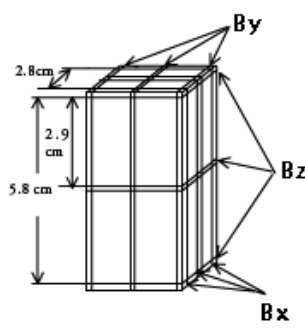

a)

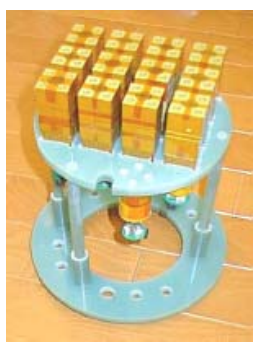

b)
Fig. 1 A 3D second order gradiometer.

The typical multichannel systems used in MCG are planar systems, which can measure only the field perpendicular to the body surface (normal component). The system developed at the TDU is able to record simultaneously both the normal and tangential components. The test subjects underwent the same measurement protocol ${ }^{6}$ ): the rest data are recorded for 4 minutes, the exercise test is performed until the target heart rate is reached and the exercise is stopped, and then immediately afterwards the stress data are measured. Rest and stress data are digitized with a sampling frequency of $1 \mathrm{kHz}$, and 48 measurement points are considered (four scanning, fixed circle), as 
shown in Fig. 2, so that the chest area is completely covered.

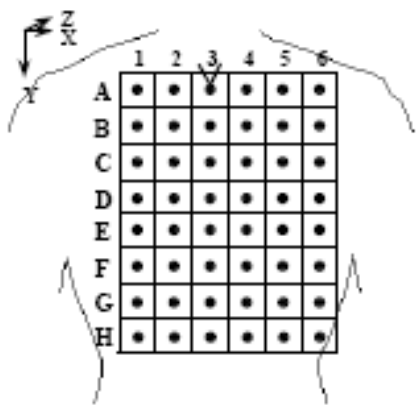

Fig. 2 Coordinate system for MCG. The coordinates from $\mathrm{A}$ to $\mathrm{H}$ and 1 to 6 represents the measurement positions.

\section{Torso Model}

The torso model chosen to solve the inverse problem is the boundary element method (BEM), that considers a piecewise homogeneous volume conductor with constant potential approximation, so that the potential inside the volume and the magnetic field outside are completely determined by the potential at the interfaces of the regions with different conductivities ${ }^{7)-8}$ ). The effect of the discontinuities is reflected in the genesis of an equivalent surface current distribution at the interfaces, also called "secondary current" to be distinguished from the primary currents, which are the biological ones. The developed model is shown in Fig. 3, and considers four compartments: torso, heart and two lungs, obtained from a CT data acquisition. The same algorithm is used to generate a triangular mesh of all compartments: first a segmentation was performed to locate a set of scattered points that correspond to a compartment surface (or volume). Then a triangulated sphere, with a defined number of points, was positioned in the center of mass of the volume to be modeled and was subsequently expanded, deforming and adapting its shape when it start finding the points of the compartment, until all points are included in the surface. The resulting model has 295 points and 586 triangular elements for each surface.

In MCG studies, a suitable description for the current source is a distributed source model, that considers the heart activation determined by an elevated number of current dipoles, with fixed location in the volume or on the surface of the heart, the so-called source space, and varying orientations and amplitudes. Such a model is a more realistic representation than single or multiple dipole models, since the heart electric activation is not localized in a small portion of the myocardium volume, but is spread as an activation wavefront. The source model used has 586 dipoles, each one positioned in one of the 586 triangles the source space surface. For every dipole only the three parameters of its momentum had to be estimated, but only 48 measurement points were available, that correspond to 144 values when the vector data were used. Assuming that $M$ is the number of magnetic field values available and $\mathrm{N}$ is the number of dipoles, we can use the lead field formulation to write the matrix equation of the inverse problem as in (1):

$$
[B]=[L \rrbracket J]
$$

where $\mathrm{B}$ is a vector $\mathrm{M}^{*} 1$ of the magnetic field values at the measurement points, $L$ is the $\mathrm{M}^{*} 3 \mathrm{~N}$ lead field matrix denoting the sensitivity distribution of the sensors, and $\boldsymbol{J}$ is the $3 \mathrm{~N}$ vector of dipole to be calculated.

Because of the underdetermined problem, the minimum norm estimation technique was used to find the most plausible solution ${ }^{9)-10)}$.

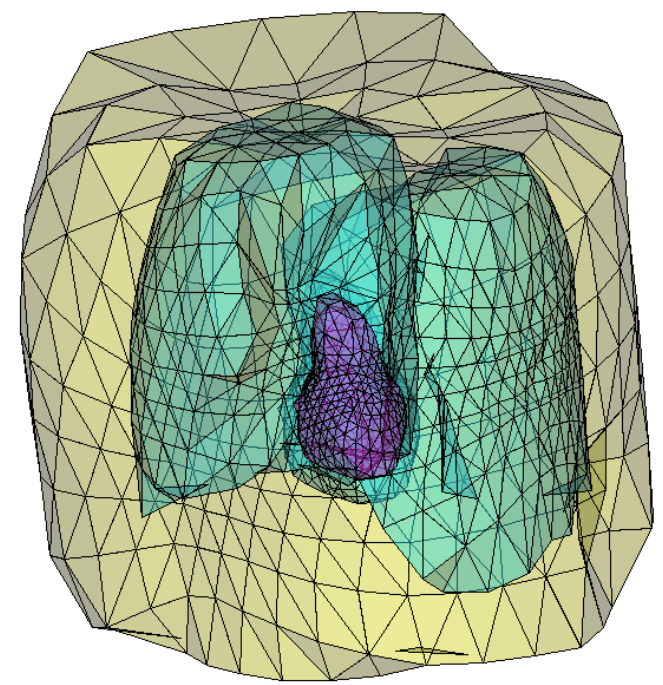

Fig. 3 BEM model consisting of 4 compartments extracted from CT data.

The conductivity values of each inhomogeneity may vary from individual to individual, and it is important to evaluate whether the contribution of a certain inhomogeneity strongly affects the total magnetic field. The values for the different conductivities were chosen in agreement with the literature ${ }^{11)-12)}$ and then were changed to test the influence of each compartment to the genesis of the field outside of the body. The typical values are $0.2 \mathrm{~S} / \mathrm{m}$ for the torso, $0.05 \mathrm{~S} / \mathrm{m}$ for the lungs and $0.6 \mathrm{~S} / \mathrm{m}$ for the heart. They appear as the underlined ones in Tab. 1. The other values were chosen to test what happens in case of the compartment is not considered (but in case of the torso, a very small value of conductivity is used, since it cannot be excluded completely), low conductivity and high conductivity for each compartment.

Three types of CSR tests were performed: first, we calculated the CSR modifying the conductivity of the torso to the very low, low and high values, and keeping fixed the ones of heart and lungs to the typical values. 
The second test was done in a similar way: the torso and lungs conductivities were fixed, whereas the heart's one was changed. Finally the CSR was performed modifying the lungs conductivity.

Table 1 Conductivity values for each compartment (S/m). The typical ones are indicated as standard. Null value for heart and lungs means that the compartment is not considered, i.e. its conductivity is the same as the torso.

\begin{tabular}{cccc}
\hline \hline & Torso & Heart & Lungs \\
\hline Standard & $\underline{0.2}$ & $\underline{0.6}$ & $\underline{0.05}$ \\
null & 0.03 & 0.2 & 0.2 \\
low & 0.1 & 0.1 & 0.01 \\
high & 0.8 & 1.4 & 0.3 \\
\hline
\end{tabular}

\section{Results}

In the analysis of stress measurements, the main time interval of interest is the ST segment. In Fig. 4 the averaged waveform of the rest and exercise data are shown. A first problem to face is that after performing the stress test, the RR interval may strongly decrease, and the corresponding time points of $\mathrm{P}, \mathrm{QRS}$ and $\mathrm{T}$ wave are different. A direct comparison of the signals on specific instants becomes rather difficult to be done since they have to be selected visually, being prone to individual errors. To avoid this problem, the two signals can be matched using a time rescaling operator, that allows to have the same time length in the segment of interest ${ }^{13)}$, as shown in Fig. 5.

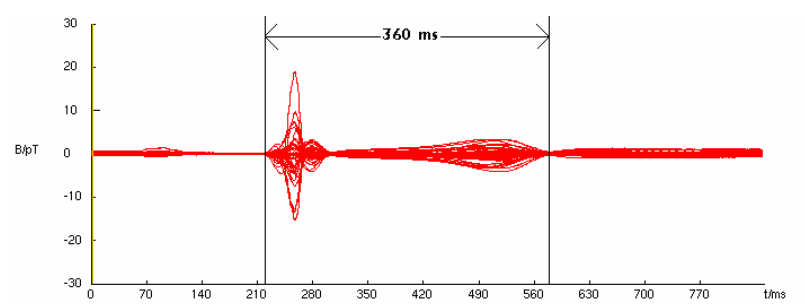

a)

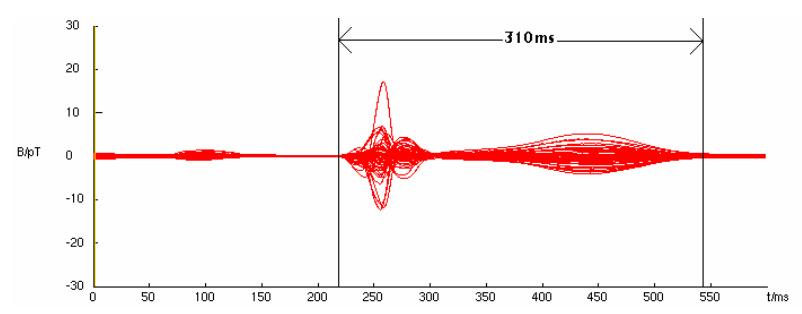

b)

Fig. 4 Rest (a) and stress (b) data before RT-match. QRST intervals and data lengths are respectively 360 $\mathrm{ms}$ and $850 \mathrm{~ms}$ in rest data and $310 \mathrm{~ms}$ and $600 \mathrm{~ms}$ in stress data.

After performing the RT-match on the averaged data, the equivalent current density was calculated as a surface current density. Such a CSR does not represent the real current flow in the heart, and the volume conductor model used is the same for all data; however, this method was applied on rest and stress data that were measured consecutively, having the test subject always the same position. The calculated CSR is then a projection of the real current flow on the heart surface, and repolarization alterations could be visualized in the ST segment.

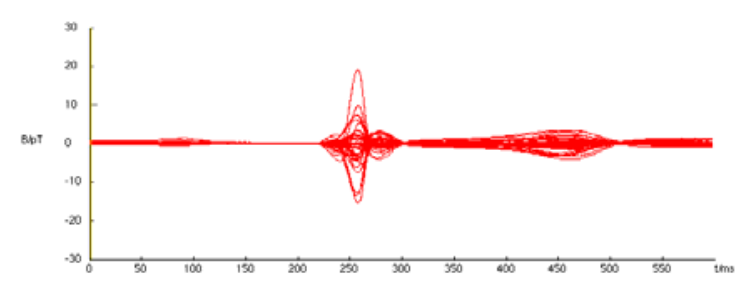

a)

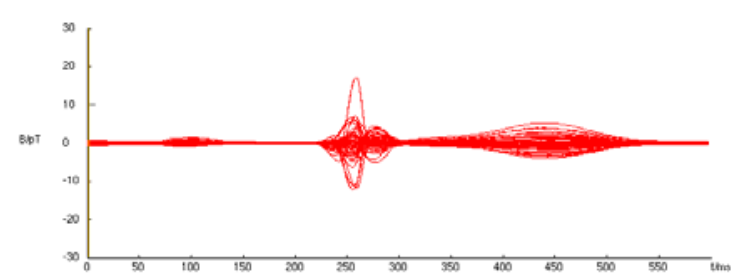

b)

Fig. 5 Rest (a) and stress (b) data after RT-match. The two data have the same time lengths and the same $\mathrm{P}$, QRS and T wave time points.

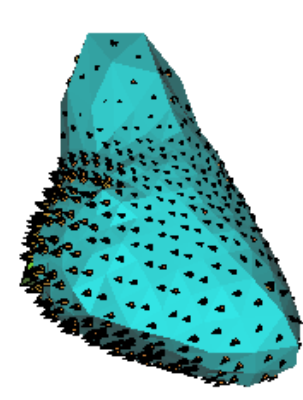

a)

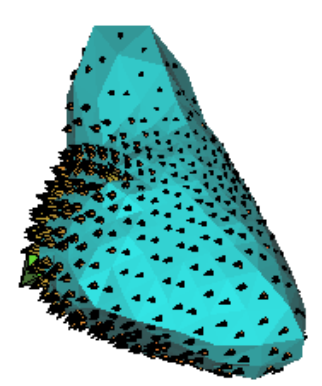

b)
Fig. 6 Rest (a) and stress (b) current sources overlaid on a triangulated modelled heart surface.

To quantify the difference in the reconstructed sources, two correlation parameters were considered using the results of the rest and exercise data. Since the source space model was the same for both data, and the dipoles had fixed positions a vector correlation value, defined in (2), can be calculated for every pair of dipoles in the same position:

$$
C_{i}^{v e c t}=\frac{\sum_{t=0}^{N_{T}}\left[\left(\vec{J}_{i}^{r}(t)-\left\langle\vec{J}_{i}^{r}\right\rangle\right)\left(\vec{J}_{i}^{s}(t)-\left\langle\vec{J}_{i}^{s}\right\rangle\right)\right]}{\sqrt{\sum_{t=0}^{N_{T}}\left(\vec{J}_{i}^{r}(t)-\left\langle\vec{J}_{i}^{r}\right\rangle\right)^{2}} \sqrt{\sum_{t=0}^{N_{T}}\left(\vec{J}_{i}^{s}(t)-\left\langle\vec{J}_{i}^{s}\right\rangle\right)^{2}}}
$$


where $\mathrm{i}$ is the dipole index, $\mathrm{N}_{\mathrm{T}}$ is length of the CSR time interval, $\vec{J}_{i}^{r}(t)$ and $\vec{J}_{i}^{s}(t)$ are respectively the dipoles of the rest and stress data, $\left\langle\vec{J}_{i}^{r}\right\rangle$ and $\left\langle\vec{J}_{i}^{s}\right\rangle$ their average value calculated over $\mathrm{N}_{T}$. Then the average value $\left\langle C^{\text {vect }}\right\rangle$ is calculated over all $C_{i}^{\text {vect }}$.

Another method to investigate the reconstructed sources is to consider an equivalent dipole calculated at each time instant, having as moment and position the values defined by (3) and (4):

$$
\begin{aligned}
& \vec{J}(t)=1 / N_{P} \sum_{i=0}^{N_{P}} \vec{J}_{i}(t) \\
& \vec{P}(t)=\frac{1}{\sum_{i=0}^{N_{P}}\left|\vec{J}_{i}(t)\right|} \sum_{i=0}^{N_{P}}\left(\left|\vec{J}_{i}(t)\right| \vec{P}_{i}\right)
\end{aligned}
$$

where $\vec{J}(t)$ is the average of all moments, $\vec{P}(t)$ is the center of mass, $i$ is the dipole index, $N_{P}$ is the number of dipoles, $\vec{J}_{i}(t)$ is the amplitude of the $\mathrm{i}$-th dipoles at the time t, and $\vec{P}_{i}$ its position. This averaged dipole represents the center of mass of the activation; once it was calculated for every time instant, a correlation coefficient that considers the shift in the dipole position, could be found in a similar way to (1) but using the equivalent average dipole as (5):

$$
C^{\text {Wvect }}=\frac{\sum_{t=0}^{N_{T}}\left[\left(\vec{J}^{r}(t)-\left\langle\vec{J}^{r}\right\rangle\right)\left(\vec{J}^{s}(t)-\left\langle\vec{J}^{s}\right\rangle\right)\left(1-\frac{\overline{P^{r}(t) P^{s}(t)}}{D_{\max }}\right)\right]}{\sqrt{\sum_{t=0}^{N_{T}}\left(\vec{J}^{r}(t)-\left\langle\vec{J}^{r}\right\rangle\right)^{2}} \sqrt{\sum_{t=0}^{N_{T}}\left(\vec{J}^{s}(t)-\left\langle\vec{J}^{s}\right\rangle\right)^{2}}}
$$

where $\overline{P^{r}(t) P^{s}(t)}$ is the distance of the two average dipoles at time $t$, and $D_{\max }$ is the maximum axis of the source space model, here used as a normalization factor.

\section{Discussion}

The CSRs are calculated for 6 test subjects. The reconstructed sources can be visualized either as surface currents as shown in Fig. 6, or using the equivalent average dipole introduced in (3) and (4). The corresponding correlation coefficients $\mathrm{C}^{\text {vect }}$ and $\mathrm{C}^{\text {Wvect }}$ were found for the tested conductivities of the different compartments. The results of $\mathrm{C}^{\text {vect }}$ are plotted in Fig. 7 to 12 in function of the conductivity, CWvect shows similar results.

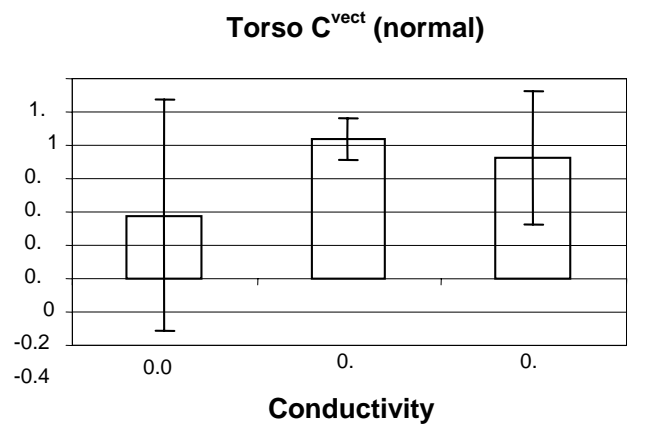

Fig. $7 \mathrm{C}^{\text {vect }}$ of normal component data for different torso conductivities.

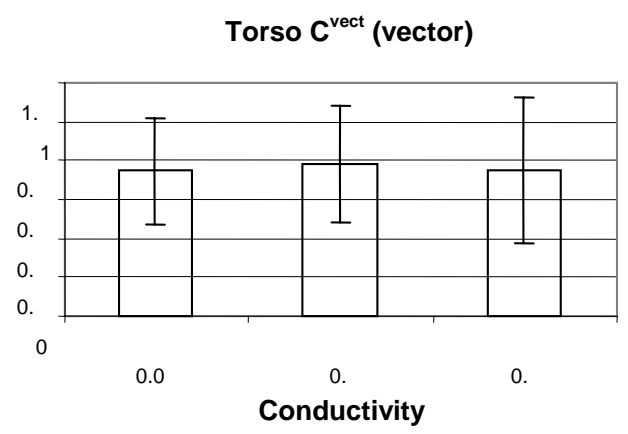

Fig. $8 \mathrm{C}^{\text {vect }}$ of vector data for different torso conductivities.

Heart $C^{\text {vect }}$ (normal)

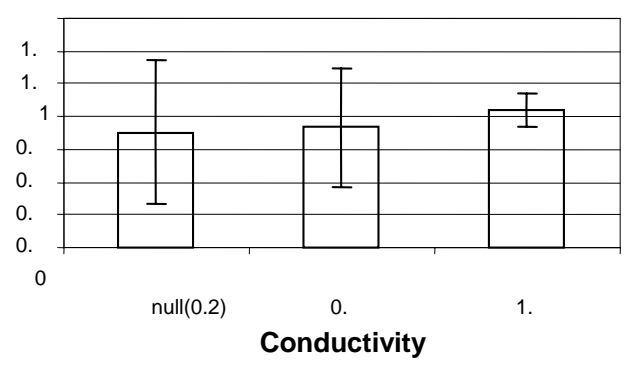

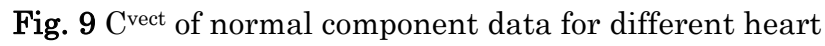
conductivities.

Heart $C^{\text {vect }}$ (vector)

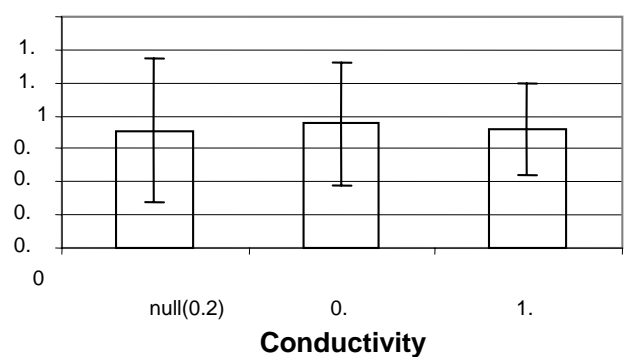

Fig. $10 \mathrm{C}^{\text {vect }}$ of vector data for different heart conductivities. 
Lungs $C^{\text {vect }}$ (normal)

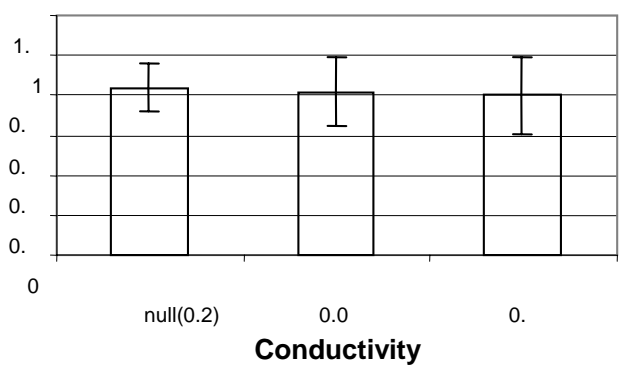

Fig. $11 \mathrm{C}^{\text {vect }}$ of normal component data for different lungs conductivities.

Lungs $C^{\text {vect }}$ (vector)

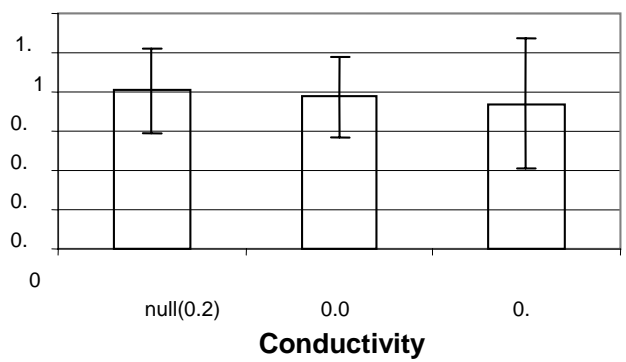

Fig. $12 \mathrm{C}^{\text {vect }}$ of vector data for different lungs conductivities.

The $\mathrm{C}^{\text {vect }}$ and $\mathrm{C}^{\text {wvect }}$ express the degree of similarity of the CSR of rest and stress data, higher values indicate small differences in the CSRs, whereas lower values indicate a greater sensitivity to induced currents that modify the ST segment ${ }^{2}$.

The results shown in Fig. 7 to 12 are summarized in Tab. 2 and 3. Among the different compartments, the variation in the lungs conductivity values had the lower effect on the coefficients values. The torso and heart conductivities showed instead a stronger influence on the CSR computation.

Table 2 Average values of $\mathrm{C}^{\text {vect }}$ for zero, low and high conductivities of each compartment in case of normal component data. Standard deviations are indicated in parentheses.

\begin{tabular}{cccc}
\hline \hline & Torso & Heart & Lungs \\
\hline null & ${ }^{*} 0.38(0.7)$ & $0.7(0.44)$ & $0.84(0.11)$ \\
low & $0.84(0.12)$ & $0.73(0.36)$ & $0.82(0.17)$ \\
high & $0.72(0.39)$ & ${ }^{* *} 0.82(0.16)$ & $0.8(0.19)$ \\
\hline
\end{tabular}

Table 3 Average values of $\mathrm{C}^{\text {vect }}$ for zero, low and high conductivities of each compartment in case of vector data. Standard deviations are indicated in parantheses.

\begin{tabular}{cccc}
\hline \hline & Torso & Heart & Lungs \\
\hline null & $* 0.74(0.27)$ & $0.71(0.43)$ & $0.8(0.21)$ \\
low & $0.78(0.3)$ & $0.75(0.37)$ & $0.77(0.2)$ \\
high & $0.74(0.37)$ & $* * 0.71(0.28)$ & $0.74(0.32)$ \\
\hline
\end{tabular}

To evaluate the differences between vector and normal component data, the $\mathrm{p}$-value of the Student t-test were calculated between the two groups of Cvect values of vector and normal data, and repeating the test for every conductivity. The most significant values were found for the lowest torso conductivity $(\mathrm{p}=0.256)^{*}$ and high heart conductivity $(\mathrm{p}=0.33)^{* *}$, where the additional information provided by the tangential components of the field showed a moderate significance for the two sets of $\mathrm{C}^{\text {vect }}$ values.

\section{Conclusion}

The effect of the volume conductor inhomogeneities were investigated through the analysis of MCG data before and after stress test. The comparison of the reconstructed sources was done using correlation coefficients, that indicate how the CSR changes on the source space surface.

The correlation coefficients calculated for the vector data looked generally less dependent on the changes of conductivities, thus suggesting that the use of a standard model of the torso volume conductor for the analysis is more reasonable than in case of planar data.

Distributed current distribution models have proven to be a promising method for non invasive analysis of stress test measurements ${ }^{14)}$. The presented method can be applied to the study of patients data to evaluate quantitatively, by means of $\mathrm{C}^{\text {vect }}$ and $\mathrm{CWvect}^{\text {, }}$ how the disturbances in the repolarization phase influence the reconstructed sources after exercise stress test.

Acknowledgements This study is a part of a scientific research program supported by a grant of the Japan Ministry of Education, Science and Culture.

\section{References}

1) K. Brockeier, S. Comani, C. Del Gratta, L. Di Donato, S. Di Luzio, A. Pasquarelli, V. Pizzella and G.L. Romani: Biomagnetism: Clinical Aspects, ed. by M.Hoke, S. N. Erné, Y. C. Hokada and G. L. Romani, 1992, pp. 509-512, (Elsevier Science, Amsterdam, 1992).

2) K. Brockmeier, S. Comani, S.N. Erné, S. Di Luzio, A. Pasquarelli, G.L. Romani: J. Electrocardiology 27, num.2, pp. 137-142, (1994).

3) C. M. Arturi, L. Di Rienzo and J. Haueisen: IEEE Trans. 
Mag. 40, num.2, pp. 631-634, (2004).

4) K. Kobayashi and Y. Uchikawa: J. Appl. Phys. 83, num. 11, pp. 6462-6464, (1998).

5) K. Kobayashi and Y. Uchikawa: Recent Advances in Biomagnetism, ed. by T. Yoshimoto, M. Kotani, S. Kuriki, H. Karibe and N. Nakasato, Tokyo, 1998, pp. 35-38 (Tohoku Univ. Press, Sendai, 1999).

6) Y. Uchikawa, B.S. Kim and K. Kobayashi: Proc. Of the $14^{\text {th }}$ Int. Conf. on Biomagnetism ed. by E. Algren, S. Ahlfors, M. Hämäläinen and D. Cohen, Boston, 2004, pp. 379-380, (Biomag, Boston, 2004).

7) F. Grynszpan and D. B. Geselowitz: J. Biophys. 13, pp. 911-925, (1973).

8) A. S. Ferguson, X. Zhang and G. Stroink: IEEE Trans. Biomed. Eng. 41, num. 5, pp. 455-460, (1994).

9) M. S. Hämäläinen and R. J. Ilmoniemi: Med. Biol. Eng.
Computing 32, pp. 35-42, (1994).

10) J. T. Nenonen, M. S. Hämäläinen and R. J. Ilmoniemi: Med. Biol. Eng. Computing 32, pp. 43-48, (1994).

11) L. A. Geddes and L. E. Baker: Med. Eng. Biol. 5, pp. 271-293, (1967)

12) S. Rush, J. A. Abildskov and R. McFee: Circ. Res. 12, pp. 40-50, (1963).

13) M. De Melis, D. Di Pietro Paolo, W. Tedeschi, M. Görnig and S.N. Erné: Int. Congr. Ser., doi:10.1016/j.ics.2007.01.016, (2007).

14) M. Görnig, M. De Melis, D. Di Pietro Paolo, W. Tedeschi, M. Liehr, H. R. Figulla and S.N. Erné: Anatolian. J. Card. 7, num. 1, pp.191-192 (2007).

Received Oct. 04, 2007; Revised Jan. 01, 2008; Accepted Feb. 14, 2008. 